\title{
Neuroimaging in Dementia: A Brief Review
}

\author{
Dipanjan Banerjee ${ }^{1,2}$, Abilash Muralidharan ${ }^{3,4}$, Abdul Rub Hakim Mohammed ${ }^{3}$, Bilal Haider Malik ${ }^{5}$ \\ 1. Neuroscience, California Institute of Behavioral Neurosciences and Psychology, Fairfield, USA 2. Geriatrics, Queen's \\ Medical Center, Nottingham University Hospitals NHS Trust, Nottingham, GBR 3. Internal Medicine, California \\ Institute of Behavioral Neurosciences and Psychology, Fairfield, USA 4. Internal Medicine, Kiruba Hospital, \\ Coimbatore, IND 5. Internal Medicine, California Institute of Behavioral Neurosciences \& Psychology, Fairfield, USA
}

Corresponding author: Dipanjan Banerjee, dbanerjee93@gmail.com

\begin{abstract}
Dementia is a clinical syndrome that manifests itself with impairment in cognitive functions owing to various neurodegenerative etiologies causing severe disability in the older population. Although the diagnosis is largely dependent on clinical examination, biomarkers can significantly aid in early diagnosis of dementia, especially in those without any clinical evidence of neurocognitive impairment. These biomarkers can be discovered in cerebrospinal fluid (CSF) or can be assessed by neuroimaging. Our goal was to discuss and assess the role of different neuroimaging techniques in the early diagnosis of relatively common etiologies of dementia. We used PubMed as search engines to look for helpful articles; most of the sources used were peer reviewed. We discussed the utility of various neuroimaging techniques, such CT, MRI, positron emission tomography (PET) scan, and single-photon emission computed tomography (SPECT), in the diagnosis of dementia. We concluded that various modern neuroimaging techniques prove to be very helpful in early identification, diagnosis, and differentiation between subtypes. However, the actual clinical utility of these tests in terms of their cost-effectivity and availability remains to be seen. Ongoing research is required to further develop biomarkers for early identification and monitor the progression of different etiologies of dementia.
\end{abstract}

Received 01/04/2020 Review began 05/27/2020 Review ended 06/07/2020 Published 06/18/2020

๑) Copyright 2020 Banerjee et al. This is an open access article distributed under the terms of the Creative Commons Attribution License CC-BY 4.0., which permits unrestricted use, distribution, and reproduction in any medium, provided the original author and source are credited.
Categories: Neurology, Psychiatry, Radiology

Keywords: neuroimaging, dementia, spect, pet, alzheimer's disease, frontotemporal dementia, vascular dementia, dementia with lewy body

\section{Introduction And Background}

\author{
"It is a strange, sad irony that so often in the territory of a disease that robs an individual of \\ memory, caregivers are often the forgotten" - Karen Wilder
}

In 2018, dementia affected 50 million people worldwide, a figure that is predicted to increase to 152 million by 2050. In 2018, the economic effect of dementia estimated about a trillion US dollars a year, and that is forecast to double by 2030 [1]. Early diagnosis is extremely important in terms of prognosis as it opens the door to a lot of possibilities for people with dementia and their families. An early diagnosis can help by initiating early treatment, which can slow down the progression of the disease yielding better therapeutic outcomes and increased quality of life [2]. For example, in Alzheimer's disease (AD), there is a preclinical stage, which is defined as a stage of the disease where the clinical features have not fully manifested yet to satisfy the criteria of mild cognitive impairment (MCI); however, there is biomarker evidence to suggest that the disease process is currently underway [3]. Biomarkers can be defined as measurable substances in the body that act as reliable predictors and indicators of a disease process. These biomarkers make it possible to identify the disease even before its clinical manifestation; hence, recently, this topic has been subjected to a lot of research as early intervention may provide better therapeutic results [2]. Neuroimaging is extremely helpful in the early diagnosis of dementia by demonstrating the presence of these biomarkers. Structural brain imaging can provide information about the shape, position, and, more importantly volume of brain tissue. Structural imaging techniques consist of MRI and CT, with MRI having much better resolution than CT. Functional brain imaging reveals how well cells in various brain regions are working by showing how actively the cells use sugar or oxygen. These most commonly include positron emission tomography (PET) and functional MRI (fMRI). Molecular brain imaging uses radiotracers to detect specific cellular or chemical changes; these include amyloid PET and single-photon emission computed tomography (SPECT) [4]. In this review, we aim to briefly assess and summarize the utility of various neuroimaging techniques to diagnose the most commonly prevalent etiologies of dementia.

\section{Review}

\section{Method}

For our review article, we have used PubMed as our search engine and database. PRISMA (Preferred Reporting Items for Systematic Reviews and Meta-analyses) guidelines have not been followed. As inclusion criteria we selected studies that were done within the past five years specifically on humans. We excluded 
any studies done on other species or older than five years. A total of 50 articles were extracted, and most of the articles used were peer reviewed. Data have been collected ethically and legally. Keywords and search results have been summarized in Table 1 .

\begin{tabular}{|c|c|c|}
\hline Keyword & Database & Results \\
\hline Neuroimaging & PubMed & 42,780 \\
\hline Dementia & PubMed & 38,726 \\
\hline SPECT & PubMed & 5,151 \\
\hline Frontotemporal dementia & PubMed & 2,269 \\
\hline PET scan & PubMed & 22,319 \\
\hline Dementia with Lewy bodies & PubMed & 1,631 \\
\hline Alzheimer's disease & PubMed & 26,716 \\
\hline Vascular dementia & PubMed & 2,472 \\
\hline ABLE 1: Keywords & & \\
\hline
\end{tabular}

\section{Discussion}

Dementia and Its Etiologies

Dementia is a common neurodegenerative illness affecting large volumes of older populations globally, which is characterized by a functional decline of different cognitive domains. Diagnostic and Statistical Manual of Mental Disorders, 5th edition (DSM-V) has included dementia as "neurocognitive disorder (NCD)" and has classified according to its etiologies and severity (mild, moderate, or severe according to the daily limitation of activities). A few examples of etiologies include $\mathrm{AD}$, vascular dementia (VaD), frontotemporal dementia (FTD), dementia due to Lewy body (DLB), and dementia due to Parkinson's disease [5]. Out of numerous etiologies linked to neurocognitive disorders, $\mathrm{AD}$ is the most common one so far; other common etiologies being VaD, FTD, and DLB [6-8].

\section{Alzheimer's Disease}

$\mathrm{AD}$ progression can be divided into three forms. Preclinical $\mathrm{AD}$, where the individual is cognitively normal but has positive biomarkers. $\mathrm{MCI}$ due to $\mathrm{AD}$, where there is evidence of declining cognitive functions, yet they do not satisfy the criteria of $\mathrm{AD}$ dementia. $\mathrm{AD}$ dementia, where the patients satisfy the criteria of diagnosis of NCD due to $\mathrm{AD}[3,9]$. The pathogenesis in $\mathrm{AD}$ can be linked to the accumulation of amyloid-beta and the development of neurofibrillary tangles [10]. The process progresses over decades before the onset of dementia. These amyloid plaques can be visible in PET scan using 11C-labeled Pittsburgh Compound B (PiB) as radiotracer [11]. The deposits were found to be localized in neocortical brain regions, including the hippocampus $[12,13]$. These deposits can be demonstrated even in individuals with MCI due to $\mathrm{AD}$ and preclinical AD. However, various studies suggest that amyloid-beta deposits can be found in the elderly population who are cognitively normal, which highlights the fact that PiB-PET scan identifying the presence of amyloid deposition can be very sensitive, yet it is not specific [14,15]. 18-Fluorodeoxyglucose (FDG) PET scan in the resting state, looking at metabolic activity of the brain, is able to highlight areas of hypometabolism in temporal and parietal regions of the cortex including posterior cingulate region in patients with AD dementia which correlates well with the areas with amyloid deposition in the PiB-PET scan $[16,17]$. However, few studies showed PiB-PET positive, but cognitively normal individuals (suspected preclinical $\mathrm{AD}$ ) did not demonstrate any metabolic abnormality in FDG-PET, although more research is required [18]. SPECT scan with 99mTc-hexamethylpropyleneamnine oxime, most commonly used to study cerebral perfusion, also shows bilateral temporoparietal hypoperfusion in $\mathrm{AD}$ patients (although can be asymmetric at times), a finding that can also be helpful in differentiating between other etiologies of dementia, such as FTD or VaD $[19,20]$. fMRI shows decreased neuronal activity in the medial temporal lobe in $\mathrm{AD}$ patients and may prove to be very useful in early diagnosis [21]. MRI with diffusion tensor imaging (DTI), which analyses white matter tracts, shows impairment of fibers connecting the hippocampus and posterior cingulate gyrus [21]. Structural scans such as MRI can identify volumetric changes in AD patients with dementia, $\mathrm{MCI}$ due to $\mathrm{AD}$, and in preclinical $\mathrm{AD}$. MRI can show increased gray matter atrophy predominantly in the hippocampus and cingulate cortex, which correlated well with the areas of amyloid deposition as seen in amyloid PET scan [21]. In summary, various neuroimaging techniques are able to 


\section{Cureus}

demonstrate signs of disease activity in $\mathrm{AD}$, including individuals with $\mathrm{MCI}$ due to $\mathrm{AD}$ and preclinical $\mathrm{AD}$, which makes them valuable for early detection of $\mathrm{AD}$.

\section{Frontotemporal Dementia}

FTD is a heterogeneous neurodegenerative disorder, which is also a fairly common cause of early-onset dementia and encompasses three clinical syndromes, out of which the most common ones are behavioral variant (bvFTD) and three language variants (semantic, non-fluent, and lopogenic) [5,22]. 3D T1 MRI shows volumetric changes in bvFTD primarily in the frontal and temporal lobes. Several studies have been able to attribute the changes to the prefrontal cortex, anterior temporal regions, the insula, anterior cingulate, and striatum, which is distinct from changes seen in the regions primarily affected in $\mathrm{AD}$ and hence helpful in differentiating between FTD and AD [23,24]. Structural imaging in patients with semantic variant shows asymmetric atrophy in the anteroinferior temporal lobe $[25,26]$. The atrophy is most commonly located on the left side; however, in some patients, the right side can be affected by volume loss [27,28]. The earliest changes in the form of volume loss can be localized to the inferior temporal and fusiform gyri, the temporal pole, and the parahippocampal and entorhinal cortex [25,29]. Nonfluent variants again primarily show left hemispheric volume loss in the inferior frontal gyrus, dorsolateral prefrontal cortex, superior temporal gyrus, and insula, which distinguishes it from other variants in the same spectrum $[25,26,29]$. In the lopogenic variant, volume loss is localized at the left temporoparietal and posterior cingulate atrophy [26,29]. In terms of amyloid PET, most of the FTD patients are revealed to be negative for A $\beta$ deposition; however, patients with lopogenic variant may show positive findings due to their association with $\mathrm{AD}$ pathogenesis $[30,31]$. Cerebral metabolism and perfusion studies such as SPECT and 18FDG PET scan are also helpful, with PET having greater utility than SPECT [32]. PET shows areas of hypometabolism, which correlates well with areas of atrophy found in structural imaging as mentioned above [33,34]. The areas of hypometabolism in FTD as evidenced by FDG-PET scan have been summarized in Table 2 .

FTD

variant

Areas of hypometabolism seen in FDG-PET

bvFTD Medial prefrontal cortex and anterior temporal region [35,36].

Semantic Asymmetrical left hemispheric involvement, primarily localized in the inferior temporal region entorhinal and perirhinal cortex $[35,37]$.

Non-fluent Inferior frontal gyrus (primarily left hemispheric [38].

Lopogenic left frontotemporoparietal and posterior cingulate region [38].

\section{TABLE 2: Areas of hypometabolism in FTD seen in FDG-PET}

bvFTD: behavioural variant of frontotemporal dementia; FTD: frontotemporal dementia; FDG-PET: fludeoxyglucose positron emission tomography

A good share of patients have familial FTD due to genetic mutations [7]. Studies suggest the existence of a presymptomatic stage where volumetric changes in the gray matter of left cingulate cortex in T1-weighted imaging and white matter changes in uncinate fasciculus and genu corpus callosum in DTI-MRI can be demonstrated, which can serve as a diagnostic and prognostic biomarker [24]. To summarize, both volumetric studies and metabolic imaging provide excellent utility in terms of presymptomatic diagnosis of FTD, which helps to differentiate not only from $\mathrm{AD}$ but also helps in differentiating between different types of FTD.

Vascular Dementia

$\mathrm{VaD}$ is the second most common cause of dementia after $\mathrm{AD}$ [39]. It is often characterized by stepwise cognitive deterioration with intervening periods of stability and acute deterioration of symptoms. The pathogenesis can be attributed to vascular insults in large vessels or multiple small vessels over a prolonged period of time [40]. Hyperintense signal on T2-weighted and fluid-attenuated inversion recovery (FLAIR) images signifies the vascular insult seen in both small vessel and large vessel diseases. T2/FLAIR imaging can also identify microhemorrhages seen in $\mathrm{VaD}[41,42]$.

Dementia With Lewy Body

DLB is also a very common form of dementia and may present with neurocognitive decline, visual symptoms, and parkinsonism [43]. Volumetric studies have shown DLB patients have more preserved temporal lobe, amygdala, and hippocampal volumes compared to AD patients [44]. FDG-PET shows significantly reduced uptake in the visual cortex in DLB patients [45]. Assessing dopaminergic function carries value in DLB, which 
can be done by dopaminergic transporter (DAT) scan by SPECT using (123I) FP-CIT as a radiotracer. This shows markedly decreased dopaminergic activity in DLB patients [46,47]. 123I-metaiodobenzylguanidine (MIBG) cardiac scintigraphy can be helpful in distinguishing DLB from AD; however, the utility may be limited by interference in outcome by co-existing cardiac disorders and diabetes mellitus $[48,49]$.

\section{Conclusions}

Hence, after careful review, we conclude that neuroimaging not only establishes its crucial role in the diagnosis of various etiologies of dementia but also it is proven to be exceedingly helpful in differentiating between multiple subtypes within a particular etiology. It also plays a leading role of neuroimaging in early diagnosis of dementia, which will pave the way for early initiation of treatment, thus delaying the progression of the disease by pharmacological means leading to better patient care. Some of the more sophisticated imaging techniques, although with their proven benefits, may face limited clinical utility, taking into consideration their cost or limited availability. The future focus of neuroimaging in dementia is very likely to shift towards multimodal imaging combining various metabolic, functional, and structural imaging to diagnose the condition, predict the progression, and monitor therapeutic benefits.

\section{Additional Information \\ Disclosures}

Conflicts of interest: In compliance with the ICMJE uniform disclosure form, all authors declare the following: Payment/services info: All authors have declared that no financial support was received from any organization for the submitted work. Financial relationships: All authors have declared that they have no financial relationships at present or within the previous three years with any organizations that might have an interest in the submitted work. Other relationships: All authors have declared that there are no other relationships or activities that could appear to have influenced the submitted work.

\section{References}

1. Patterson C: World Alzheimer Report 2018. Alzheimer's Disease International (ADI)), London, UK; 2018.

2. Diagnostic criteria for dementia. (2018). Accessed: December 28, 2019: https://www.dementia.org.au/files/helpsheets/Helpsheet-DementiaQandA11DiagnosticCriteriaForDementia_english.pdf.

3. Dubois B, Hampel H, Feldman HH, et al.: Preclinical Alzheimer's disease: definition, natural history, and diagnostic criteria. Alzheimer's Dement. 2016, 12:292-323. 10.1016/j.jalz.2016.02.002

4. Tartaglia MC, Rosen H: Neuroimaging in dementia. The Behavioral Neurology of Dementia. Miller BL, Boeve B F (ed): Cambridge University Press, Cambridge; 2009. 101-119. 10.1017/CBO9780511581410.008

5. American Psychiatric Association: Neurocognitive disorders. Diagnostic and Statistical Manual of Mental Disorders, 5th Edition. American Psychiatric Association, Arlington, VA; 2013.

6. World Health Organisation. Dementia factsheet . (2019). Accessed: December 26, 2019 : https://www.who.int/news-room/fact-sheets/detail/dementia.

7. Onyike CU, Diehl-Schmid J: The epidemiology of frontotemporal dementia. Int Rev Psychiatry. 2013, 25:130-137. 10.3109/09540261.2013.776523

8. Hébert R, Brayne C: Epidemiology of vascular dementia. Neuroepidemiology. 1995, 14:240-257. 10.1159/000109800

9. Dubois B, Feldman HH, Jacova C, et al.: Revising the definition of Alzheimer's disease: a new lexicon . Lancet Neurol. 2010, 9:1118-1127. 10.1016/\$1474-4422(10)70223-4

10. Querfurth HW, LaFerla FM: Mechanisms of disease Alzheimer's disease . N Engl J Med. 2010, 362:329-344. 10.1056/NEJMra0909142

11. Klunk WE, Engler H, Nordberg A, et al.: Imaging brain amyloid in Alzheimer's disease with Pittsburgh Compound-B. Ann Neurol. 2004, 55:306-319. 10.1002/ana.20009

12. Thal DR, Rüb U, Orantes M, Braak H: Phases of A -deposition in the human brain and its relevance for the development of AD. Neurology. 2002, 58:1791-1800. 10.1212/WNL.58.12.1791

13. Ewers M, Sperling RA, Klunk WE, Weiner MW, Hampel H: Neuroimaging markers for the prediction and early diagnosis of Alzheimer's disease dementia. Trends Neurosci. 2011, 34:430-442. 10.1016/j.tins.2011.05.005

14. Bennett DA, Schneider JA, Arvanitakis Z, et al.: Neuropathology of older persons without cognitive impairment from two community-based studies. Neurology. 2006, 66:1837-1844. 10.1212/01.wnl.0000219668.47116.e6

15. Pike KE, Savage G, Villemagne VL, et al.: $\quad \beta$-amyloid imaging and memory in non-demented individuals: evidence for preclinical Alzheimer's disease. Brain. 2007, 130:2837-2844. 10.1093/brain/awm238

16. Silverman DHS: Brain 18F-FDG PET in the diagnosis of neurodegenerative dementias: comparison with perfusion SPECT and with clinical evaluations lacking nuclear imaging. J Nucl Med. 2004, 45:594-607.

17. Edison P, Archer HA, Hinz R, et al.: Amyloid, hypometabolism, and cognition in alzheimer disease: an [11C]PIB and [18F]FDG PET study. Neurology. 2007, 68:501-508. 10.1212/01.wnl.0000244749.20056.d4

18. Cohen AD, Price JC, Weissfeld LA, et al.: Basal cerebral metabolism may modulate the cognitive effects of $A \beta$ in mild cognitive impairment: an example of brain reserve. J Neurosci. 2009, 29:14770-14778. 10.1523/JNEUROSCI.3669-09.2009

19. Devous MD: Functional brain imaging in the dementias: role in early detection, differential diagnosis, and longitudinal studies. Eur J Nucl Med Mol Imaging. 2002, 29:1685-1696. 10.1007/s00259-002-0967-2

20. Silverman DHS, Small GW, Phelps ME: Clinical value of neuroimaging in the diagnosis of dementia: sensitivity and specificity of regional cerebral metabolic and other parameters for early identification of 
Alzheimer’s disease. Clin Positron Imaging. 1999, 2:119-130. 10.1016/s1095-0397(99)00020-5

21. Rombouts SARB, Barkhof F, Veltman DJ, et al.: Functional MR imaging in Alzheimer's disease during memory encoding. Am J Neuroradiol. 2000, 21:1869-1875.

22. Seelaar H, Rohrer JD, Pijnenburg YAL, Fox NC, Van Swieten JC: Clinical, genetic and pathological heterogeneity of frontotemporal dementia: a review. J Neurol Neurosurg Psychiatry. 2011, 82:476-486. 10.1136/jnnp.2010.212225

23. Schroeter ML, Laird AR, Chwiesko C, et al.: Conceptualizing neuropsychiatric diseases with multimodal data-driven meta-analyses: the case of behavioral variant frontotemporal dementia. Cortex. 2014, 57:22-37. 10.1016/j.cortex.2014.02.022

24. Jiskoot LC, Panman JL, Meeter LH, et al.: Longitudinal multimodal MRI as prognostic and diagnostic biomarker in presymptomatic familial frontotemporal dementia. Brain. 2019, 142:193-208. 10.1093/brain/awy288

25. Rohrer JD, Warren JD, Modat M, et al.: Patterns of cortical thinning in the language variants of frontotemporal lobar degeneration. Neurology. 2009, 72:1562-1569. 10.1212/WNL.0b013e3181a4124e

26. Gorno-Tempini ML, Dronkers NF, Rankin KP, et al.: Cognition and anatomy in three variants of primany progressive aphasia. Ann Neurol. 2004, 55:335-346. 10.1002/ana.10825

27. Whitwell JL, Przybelski SA, Weigand SD, et al.: Distinct anatomical subtypes of the behavioural variant of frontotemporal dementia: a cluster analysis study. Brain. 2009, 132:2932-2946. 10.1093/brain/awp232

28. Chan D, Fox NC, Jenkins R, Scahill RI, Crum WR, Rossor MN: Rates of global and regional cerebral atrophy in AD and frontotemporal dementia. Neurology. 2001, 57:1756-1763. 10.1212/wnl.57.10.1756

29. Rogalski E, Cobia D, Harrison TM, Wieneke C, Weintraub S, Mesulam MM: Progression of language decline and cortical atrophy in subtypes of primary progressive aphasia. Neurology. 2011, 76:1804-1810. 10.1212/WNL.0b013e31821ccd3c

30. Drzezga A, Grimmer T, Henriksen G, et al.: Imaging of amyloid plaques and cerebral glucose metabolism in semantic dementia and Alzheimer's disease. Neuroimage. 2008, 39:619-633. 10.1016/j.neuroimage.2007.09.020

31. Engler H, Santillo AF, Wang SX, et al.: In vivo amyloid imaging with PET in frontotemporal dementia . Eur J Nucl Med Mol Imaging. 2008, 35:100-106. 10.1007/s00259-007-0523-1

32. Davison CM, O’Brien JT: A comparison of FDG-PET and blood flow SPECT in the diagnosis of neurodegenerative dementias: a systematic review. Int J Geriatr Psychiatry. 2014, 29:551-561. 10.1002/gps.4036

33. Foster NL, Heidebrink JL, Clark CM, et al.: FDG-PET improves accuracy in distinguishing frontotemporal dementia and Alzheimer's disease. Brain. 2007, 130:2616-2635. 10.1093/brain/awm177

34. Matias-Guiu JA, Cabrera-Martín MN, García-Ramos R, et al.: Evaluation of the new consensus criteria for the diagnosis of primary progressive aphasia using fluorodeoxyglucose positron emission tomography. Dement Geriatr Cogn Disord. 2014, 38:147-152. 10.1159/000358233

35. Diehl-Schmid J, Grimmer T, Drzezga A, et al.: Longitudinal changes of cerebral glucose metabolism in semantic dementia. Dement Geriatr Cogn Disord. 2006, 22:346-351. 10.1159/000095624

36. Verfaillie SCJ, Adriaanse SM, Binnewijzend MAA, et al.: Cerebral perfusion and glucose metabolism in Alzheimer's disease and frontotemporal dementia: two sides of the same coin?. Eur Radiol. 2015, 25:30503059. 10.1007/s00330-015-3696-1

37. Desgranges B, Matuszewski V, Piolino P, et al.: Anatomical and functional alterations in semantic dementia: a voxel-based MRI and PET study. Neurobiol Aging. 2007, 28:1904-1913.

10.1016/j.neurobiolaging.2006.08.006

38. Aizenstein HJ, Nebes RD, Saxton JA, et al.: Frequent amyloid deposition without significant cognitive impairment among the elderly. Arch Neurol. 2008, 65:1509-1517. 10.1001/archneur.65.11.1509

39. Cassel CK, Ek K: Demography and epidemiology of age-associated neuronal Impairment . Functional Neurobiology of Aging. Hof P, Mobbs C (ed): Academic Press, San Diego; 2001. 31-50. 10.1016/b978012351830-9/50006-8

40. Falk WE, Wiechers IR: Demented patients. Massachusetts General Hospital Handbook of General Hospital Psychiatry (Sixth Edition). Stern TA, Freudenreich GL, Smith F, Fricchione G, Rosenbaum J (ed): Saunders, Philadelphia, PA; 2010. 105-118. 10.1016/b978-1-4377-1927-7.00011-X

41. Cordonnier C, Van Der Flier WM, Sluimer JD, Leys D, Barkhof F, Scheltens P: Prevalence and severity of microbleeds in a memory clinic setting. Neurology. 2006, 66:1356-1360. 10.1212/01.wnl.0000210535.20297.ae

42. Chui HC: Subcortical ischemic vascular dementia. Neurol Clin. 2007, 25:717-740. 10.1016/j.ncl.2007.04.003

43. Emre M, Aarsland D, Brown R, et al.: Clinical diagnostic criteria for dementia associated with Parkinson's disease. Mov Disord. 2007, 22:1689-1707. 10.1002/mds.21507

44. Barber R, Gholkar A, Scheltens P, Ballard C, McKeith IG, O’Brien JT: MRI volumetric correlates of white matter lesions in dementia with Lewy bodies and Alzheimer's disease. Int J Geriatr Psychiatry. 2000, 15:911916. 10.1002/1099-1166(200010)15:10<911::aid-gps217>3.0.co;2-t

45. Gupta V, Verma R, Ranjan R, et al.: Metabolic imaging patterns in posterior cortical atrophy and Lewy body dementia. Nucl Med Commun. 2019, 40:1275-1282. 10.1097/MNM.0000000000001102

46. O'Brien JT, Colloby S, Fenwick J, et al.: Dopamine transporter loss visualized with FP-CIT SPECT in the differential diagnosis of dementia with Lewy bodies. Arch Neurol. 2004, 61:919-925. 10.1001/archneur.61.6.919

47. Brigo F, Turri G, Tinazzi M: 123I-FP-CIT SPECT in the differential diagnosis between dementia with Lewy bodies and other dementias. J Neurol Sci. 2015, 359:161-171. 10.1016/j.jns.2015.11.004

48. King AE, Mintz J, Royall DR: Meta-analysis of 123I-MIBG cardiac scintigraphy for the diagnosis of Lewy body-related disorders. Mov Disord. 2011, 26:1218-1224. 10.1002/mds.23659

49. Williams M, Rodnight R: Evidence for a role for protein phosphorylation in synaptic function in the cerebral cortex mediated through a $\beta$-noradrenergic receptor. Brain Res. 1974, 77:502-506. 10.1016/00068993(74)90639-8 